\section{Therapie bei NHL im Praxistest}

\begin{abstract}
Bei verschiedenen lymphatischen Malignomen hat sich Bendamustin in Monotherapie oder in Kombination mit Rituximab als wirksame und relativ gut tolerierte Therapie etabliert. Ob das auch in der täglichen Routine so ist, wurde in einer nichtinterventionellen Studie geprüft.
\end{abstract}

$\mathrm{D}$ azu wurden zwischen April 2010 und Oktober 2011 in 57 deutschen Zentren Daten zur Therapie von $324 \mathrm{~Pa}$ tienten mit fortgeschrittenen indolenten Non-Hodgkin-Lymphomen (NHL) und Mantelzell-Lymphomen (MCL) erhoben, die als Erstlinientherapie ein vom jeweiligen betreuenden Arzt zu wählendes Regime mit Bendamustin erhielten. Beurteilt wurde das beste Ansprechen, das die bei Therapiebeginn im Mittel 69 Jahre alten Patienten erreichten.

94\% der Patienten erhielten Bendamustin in Kombination mit Rituximab mit einer medianen Bendamustin-Dosis von $177 \mathrm{mg} / \mathrm{m}^{2}$ pro Zyklus. Die Dosierung $\left(88 \mathrm{mg} / \mathrm{m}^{2}\right.$ meist an den Tagen 1 und 2 eines 4-wöchigen Zyklus) entsprach damit überwiegend der in Leitlinien empfohlenen und in einer PhaseIII-Studie belegten Dosierung in Kombination mit Rituximab. Im Median erhielten die Patienten 6 Zyklen dieser Therapie. $5 \%$ wurden mit einer Bendamustin-Monotherapie und die übrigen mit anderen Bendamustin-Kombinationen behandelt. Für 281 Patienten konnte das Ansprechen ausgewertet werden.

Die Gesamtansprechrate lag bei $86 \%$ - mit $43 \%$ kompletten Remissionen, bei $10 \%$ der Patienten war die Erkrankung stabil, bei $4 \%$ progredient. Weil die Studie wegen Änderung der Zulassung vorzeitig beendet werden musste, konnte die Langzeiteffektivität nicht beurteilt werden.

Nebenwirkungen aller Schweregrade traten bei $50 \%$ der Patienten auf - wahrscheinlich seien solche geringerer Schwere nicht vollständig berichtet worden, so die Forscher. Am häufigsten waren hämatologische Nebenwirkungen (35\%). $17 \%$ der Patienten wiesen Toxizitäten vom Grad 3 (15\%) und 4 (2\%) auf, $2 \mathrm{~Pa}$ tienten auch solche vom Grad 5.

Fazit: Eine Bendamustin-basierte Erstlinientherapie von Patienten mit einem fortgeschrittenen indolenten NHL oder MCL ist in der klinischen Routine wirksam und wird gut toleriert. Die Ansprechraten sind denen in interventionellen klinischen Studien vergleichbar.

Friederike Klein

Becker $\mathrm{M}$ et al. Bendamustine as first-line treatment in patients with advanced indolent non-Hodgkin lymphoma and mantle cell lymphoma in German routine clinical practice. Ann Hematol. 2015;94(9):1553-8.

\section{Behandlung und immunologische Störungen beeinflussen Melanomrisiko beim NHL}

\author{
Überlebende eines Non-Hodgkin-Lymphoms (NHL) leben mit der Gefahr, \\ kutane Melanome zu entwickeln. Bisher ist nicht klar, wie sich das Risiko je \\ nach Behandlung und immunologischen Risikofaktoren unterscheidet.
}

\begin{abstract}
$\mathrm{n}$ einer aktuellen Studie wurden Daten zu sekundären Melanomen in einer Kohorte von 44.870 Patienten aus dem SEER-Register analysiert, bei denen zwischen 1992 und $2009 \mathrm{im}$ Alter von
\end{abstract}

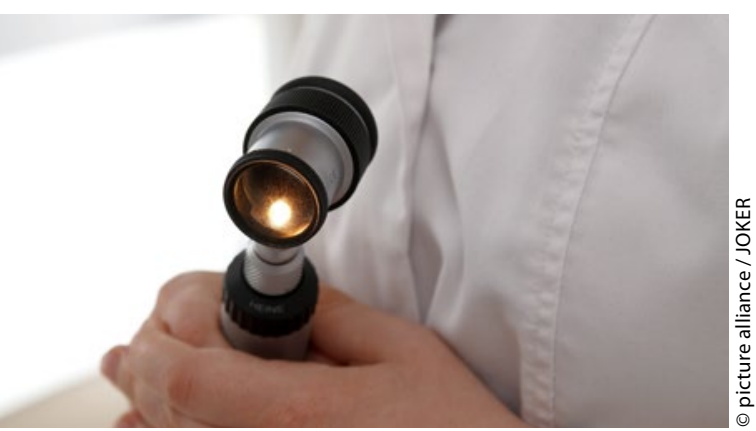

Bei Lymphom-Überlebenden besonders auf Hautveränderungen achten!
66 - 83 Jahren erstmals ein NHL diagnostiziert worden war. Alle Patienten hatten nach der Erstdiagnose mindestens ein Jahr überlebt.

Insgesamt traten in der Kohorte 202 sekundäre Melanome auf, davon 91 nach chronischer lymphatischer Leukämie/ kleinzelligem B-Zell-Lymphom (CLL/ SLL) und 111 nach einem anderen NHLSubtyp. Die kumulative Inzidenz bis zu einem Alter von 85 Jahren betrug bei CLL/SLL $1,37 \%$, bei den anderen Subtypen $0,78 \%$. Signifikant erhöht war das Melanom-Risiko bei Patienten, die eine Fludarabin-haltige Chemotherapie erhalten hatten $(n=18)$. Hier betrug die Hazard Ratio bei Therapie mit Rituximab $(\mathrm{n}=8) 1,92(95 \%$-Konfidenzintervall $[95 \%-\mathrm{KI}] 1,09-3,40)$, ohne Rituximab $(\mathrm{n}=10) 2,92(95 \%$-KI 1,42-6,01).
Bei Patienten mit CLL/SLL erhöhten T-Zell-aktivierende Autoimmunerkrankungen das Melanom-Risiko zusätzlich um einen Faktor zwischen 2,27 und 2,92. Bei anderen NHL-Subgruppen zeigte sich eine solche Assoziation mit T-Zelloder B-Zell-vermittelten Autoimmunerkrankungen nicht. Infektionen waren allgemein nicht mit einer erhöhten Melanom-Rate assoziiert, es gab aber Assoziationen zu Harnwegsinfektionen bei CLL/SLL und zu lokaler Sklerodermie, Pneumonie und gastrohepatischen Infekten bei anderen NHL.

Fazit: Die Ergebnisse deuten darauf hin, dass Fludarabin-haltige Regimes bei allen NHL, und immunologische Störungen bei CLL/SLL zur Entwicklung von sekundären Melanomen beitragen. Die Ergebnisse unterstreichen die Bedeutung einer hohen Wachsamkeit bei Überlebenden eines NHL, um eine Früherkennung von Sekundärmelanomen zu ermöglichen. Friederike Klein

Lam CJK et al. Risk Factors for Melanoma Among Survivors of Non-Hodgkin Lymphoma. J Clin Oncol. 2015;33(28):3096-104. 